\title{
DOS ESPECIES NOTABLES DE PHORADENDRON (VISCACEAE) DE LA MIXTECA OAXAQUEÑA (MÉXICO), UNA NUEVA Y UNA COMPLEMENTADA*
}

\author{
Jerzy RzedowsKi ${ }^{1}$ y Graciela Calderón de Rzedowski** \\ Instituto de Ecología, A.C., Centro Regional del Bajío, \\ Apdo. postal 386, 61600 Pátzcuaro, Mich., México. \\ 1Autor para la correspondencia: jerzy.rzedowski@inecol.edu.mx
}

\section{RESUMEN}

Se describe como nuevo y se ilustra a Phoradendron perredactum, un parásito de ramas de Bursera discolor, planta diminuta, carente de hojas y también prácticamente de tallo. De ésta se observaron dos tipos de individuos, los más comunes ramificados, de menos de $1 \mathrm{~cm}$ de largo y con prevalencia de flores femeninas, y otros escasos, por lo general solitarios, hasta de $3 \mathrm{~cm}$ de largo, mayormente con flores masculinas. Se complementa asimismo la descripción de P. olae Kuijt con datos relativos a plantas masculinas y a hospederos conocidos. Ambas especies conviven en el distrito de Huajuapan de León, en el noroeste del estado de Oaxaca, México.

Palabras clave: México, Oaxaca, Phoradendron, planta parásita, Viscaceae.

\footnotetext{
* Trabajo realizado con apoyo del Instituto de Ecología, A.C. (cuenta 20006), del Consejo Nacional de Ciencia y Tecnología y de la Comisión Nacional para el Conocimiento y Uso de la Biodiversidad.

** Los autores agradecen a la Dra. Lourdes Rico por la identificación de los hospederos de Phoradendron olae y al Dr. Victor W. Steinmann por la revisión del texto del resumen en inglés.
} 


\section{ABSTRACT}

Phoradendron perredactum, a miniature leafless and practically acaulescent stem parasite of Bursera discolor, is described as new and illustrated. Two types of individuals were observed: the more common branched ones, less than $1 \mathrm{~cm}$ long with a prevalence of female flowers, and the scarcer usually solitary ones to $3 \mathrm{~cm}$ long, with a prevalence of male flowers. The description of $P$. olae Kuijt is complemented with information concerning male plants and hosts. Both plants occur sympatrically in the District of Huajuapan de León in northwestern Oaxaca, Mexico.

Key words: México, Oaxaca, Phoradendron, parasitic plant, Viscaceae.

La cuenca del río Balsas descuella por la abundancia de endemismos, muchos de ellos bastante locales y que poco a poco se van descubriendo, pues su territorio no ha sido todavía suficientemente explorado desde el punto de vista biológico. Una de sus porciones menos conocidas, sobre todo en lo que concierne a la flora, es su extremo oriental y en particular la región drenada por el tributario río Mixteco, perteneciente al sector noroccidental del estado de Oaxaca.

La vegetación de la gran parte de la comarca en cuestión corresponde al bosque tropical caducifolio que en muchos lugares ya ha sido destruido o intensamente perturbado. Sin embargo, se encuentran todavía numerosos rincones que conservan aún la biota original en forma escasamente modificada.

El tema de la presente contribución corresponde a dos especies de Phoradendron que parasitan los árboles en esta región. Ambas son aparentemente plantas poco comunes y difieren manifiestamente del grueso de las especies del género, pues una de ellas está casi desprovista de hojas, mientras que la otra no solamente es áfila, sino también prácticamente carece de tallo y es de tamaño diminuto.

La segunda no parece haber sido descrita todavía; la primera ya ha sido dada a conocer hace 14 años (Kuijt, 1997, 2003) con base en materiales colectados en un lugar aledaño del estado de Puebla. De esta última se está completando la información morfológica y ecológica obtenida de poblaciones muestreadas en Oaxaca.

Phorandendron perredactum Rzed. \& Calderón, sp.n. Fig. 1.

Fruticulus minutissimus fere acaulis aphyllus monoecius glaber; inflorescentiae (1)2-6 ab base caulinari vix perceptibile oriundae, 0.4-3 cm longae, segmen- 
tis plerumque 4-5, floribus per segmentum 2-28 in seriebus non dispositis; flores masculi trimeri antheris bilocularibus; flores feminei trimeri; fructus ovoideus vel aliquantum pyriformis, 4-5 mm longus, tepalis inapertis.

Arbustito diminuto, parásito de ramas relativamente gruesas de Bursera discolor, prácticamente carente de tallo y en consecuencia de hojas y de catafilos, esencialmente monoico, de color café-morado, glabro salvo la presencia de cilios en el margen de las brácteas; inflorescencias 1 o con más frecuencia 2 a 6 partiendo de una base caulinar apenas perceptible, de 0.4 a $3 \mathrm{~cm}$ de largo, sus segmentos por lo general 4 o 5, flores 2 a 28 por segmento, sin disponerse en hileras; flores masculinas trímeras, anteras biloculares, pistilodio diminuto; flores femeninas trímeras; fruto ovoide a algo piriforme, de 4 a $5 \mathrm{~mm}$ de largo, rugoso, café-morado, con tépalos cerrados en el ápice.

Tipo: México, Oaxaca, 10 km al NE de Mariscala, sobre el camino a Huajuapan, bosque tropical caducifolio, alt. 1350 m, 2.XI.1976, J. Rzedowski 34790 (IEB, isotipos por distribuirse).

En lo que concierne a la distribución de los sexos, cabe observar que la gran mayoría de las inflorescencias presentan tanto flores femeninas como masculinas; sin embargo, se pueden distinguir dos tipos de individuos:

los muy comunes pequeños, que sobresalen menos de $1 \mathrm{~cm}$ de la superficie del hospedero; suelen consistir de varias inflorescencias dispuestas en forma más o menos radial; estas inflorescencias tienen menos de 15 flores, entre las cuales predominan o a veces son exclusivas las femeninas;

los mucho más escasos de mayor tamaño, que llegan a medir hasta $3 \mathrm{~cm}$ de alto; consisten por lo común de una sola inflorescencia por lo general erecta con más de 100 flores, en su mayoría masculinas.

La planta es rara, únicamente se conoce de esta colecta procedente de un solo árbol y no se ha vuelto a encontrar ni siquiera en el sitio original, aunque también es factible que haya pasado inadvertida en función de su pequeñísima talla.

Su único hospedero registrado Bursera discolor fue descrita de la parte central de Guerrero y se ha colectado en la parte oriental de la cuenca del Balsas, así como en la región costera de Oaxaca; se ha citado también de los estados de Colima, México y Michoacán, pero se ha visto recientemente que los reportes en cuestión se basan en ejemplares erróneamente identificados. Este árbol es relativamente fre- 


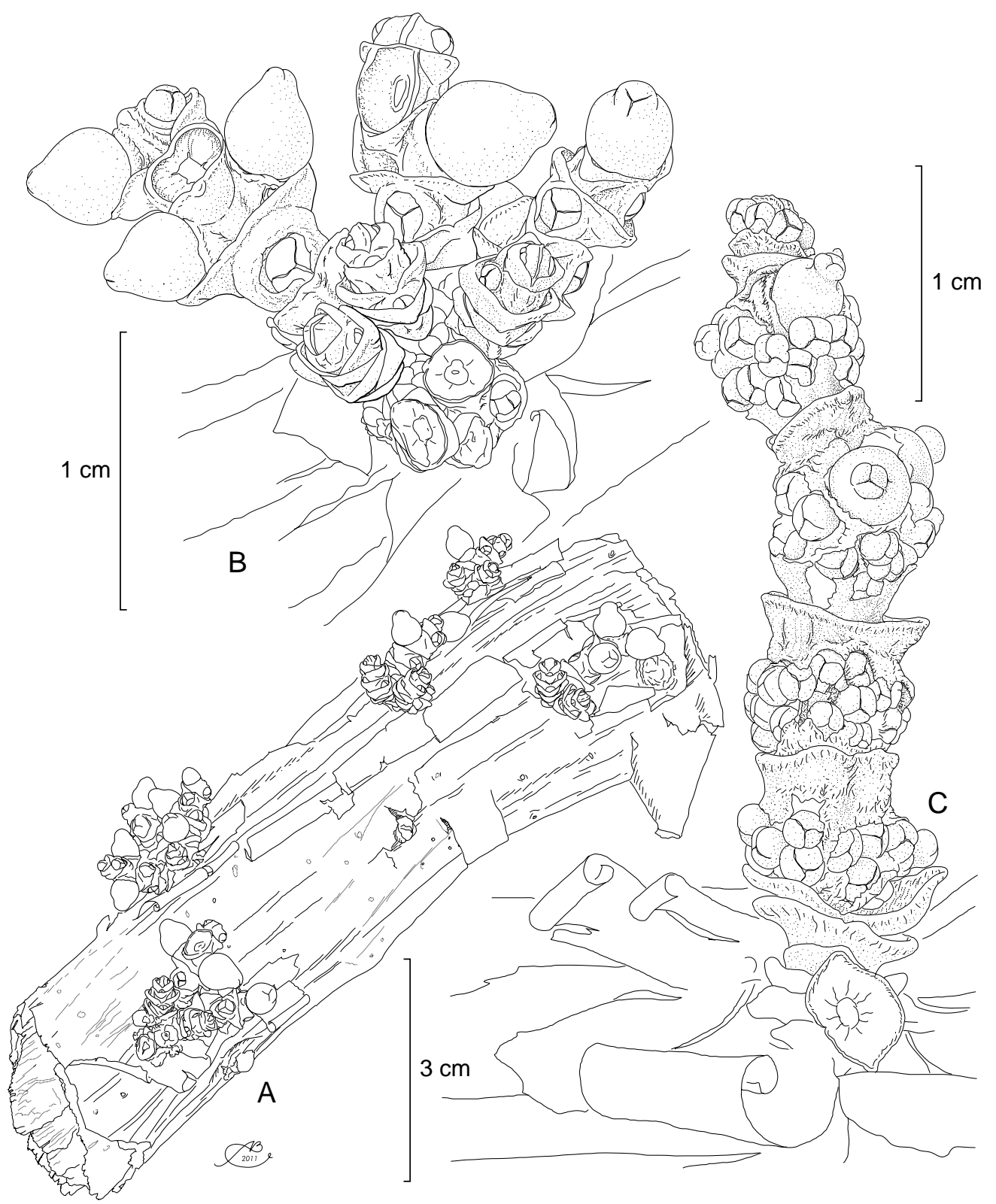

Fig. 1. A. Varios individuos proliferando sobre la rama de Bursera discolor; B. individuo pequeño y ramificado; $C$. individuo de mayor tamaño sin ramificar. Ilustrado por Alfonso Barbosa 
cuente en la subcuenca del río Mixteco y es allí donde cabría enfocar la búsqueda de otras poblaciones de la especie parásita que se está describiendo.

Phoradendron perredactum difiere de las demás especies conocidas del género en la reducción casi completa del tallo y en el tamaño diminuto de toda la planta, en la cual sólo se manifiesta en forma conspicua el haustorio y las inflorescencias. En tal hábito muestra una manifiesta analogía con Arceuthobium minutissimum J. D. Hooker, parásito de pino, conocido de los Himalayas.

En lo que concierne a sus afinidades con otros componentes del género, no se tiene una idea clara. El taxon nuevo definitivamente no parece estar relacionado con el simpátrico P. olae (ni con el probablemente cercano P. californicum Nutt.), lo que confirma la noción de que la tendencia a la reducción de las hojas se ha ido desarrollando en forma independiente y paralela en varios linajes filéticos en Phoradendron. En su condición de planta casi áfila, monoica y con inflorescencias cortas P. aphyllum Steyermark, descrito de Venezuela, se asemeja a P. perredactum, pero tales caracteres se encuentran también en varias otras especies del género no emparentadas entre sí, por lo que no hay base suficiente para definir un vínculo estrecho. Tampoco la otra especie áfila $P$. fragile Urban, conocida de Brasil, muestra rasgos que la aproximen a la última.

Un aspecto interesante que valdría la pena investigar es si, en función de la merma tan drástica de las superficies fotosintéticas, todavía existe clorofila en los tejidos de P. perredactum y si cabe considerar a la planta como un hemiparásito, como es el caso de otras especies de la familia Viscaceae.

El nombre de la especie (perredactum = sumamente reducido) alude no solamente al hecho de su diminuto tamaño, sino sobre todo a la casi total supresión de sus porciones vegetativas expuestas.

Phoradendron olae Kuijt, Brittonia 49: 185. 1997. Tipo: México, Puebla, paraje “La Tigre”, 2.5 km al NW de San Pedro Las Palmas, municipio de Jolalpan, alt. 1281 m, bosque tropical caducifolio, exposición NW, 6.I.1990, Razo y García IIIc35 (MEXU, isotipo en IEB).

Arbusto hasta de $60 \mathrm{~cm}$ de alto y de diámetro, profusamente ramificado, glabro, dioico; tallos rollizos, los jóvenes con espesa cubierta pruinosa, lo que les confiere coloración grisáceo-verdosa, los más maduros hasta de $1 \mathrm{~cm}$ de diámetro, café-grisáceos a café-negruzcos, entrenudos hasta de $4 \mathrm{~cm}$ de largo, los basales de las ramificaciones provistos de uno (o a veces dos) pares de catafilos, los de cada par parcialmente unidos entre sí, sus ápices café-rojizos, al menos en la juventud; hojas 
diminutas, por lo común de contorno triangular, de 2 a $5 \mathrm{~mm}$ de largo, agudas a acuminadas en el ápice, gruesas y carnosas, caducas; inflorescencias solitarias o dispuestas por pares en los nudos, pruinosas en la juventud, sus brácteas café-rojizas; las masculinas hasta de $3 \mathrm{~cm}$ de largo y con 3 a 6 segmentos, cada segmento con 2 a 14 flores, éstas por lo general dispuestas en 4 hileras longitudinales; las femeninas hasta de 3.5(4) cm de largo, con 3 a 5 segmentos, cada segmento con 2 flores de posición opuesta; fruto ovoide a subgloboso, hasta de $5 \mathrm{~mm}$ de diámetro, morado-rojizo en la madurez, sus tépalos cerrados.

Hasta la fecha la planta sólo se había dado a conocer de dos colectas realizadas en la localidad tipo y un lugar aledaño. Se registran ahora los siguientes ejemplares adicionales:

México, Oaxaca, $10 \mathrm{~km}$ al NE de Mariscala, sobre la carretera a Huajuapan de León, alt. 1350 m, 26.X.1979, parásito de Acacia cochliacantha, planta masculina, J. Rzedowski 36514 (IEB); planta femenina, J. Rzedowski 36516 (IEB)., $5 \mathrm{~km}$ al E de Mariscala, sobre la carretera a Huajuapan de León, alt. 1350 m, 17.II.1983, parásito de Leucaena pallida, planta masculina, J. Rzedowski 38055 (IEB); planta femenina, J. Rzedowski 38056 (IEB).

Como observación adicional cabe agregar que la especie no es frecuente ni abundante en la región de Mariscala. A su vez, los individuos masculinos son más difíciles de encontrar que los femeninos.

\section{LITERATURA CITADA}

Kuijt, J. 1997. Phoradendron olae Kuijt, a new species from Mexico pivotal in the taxonomy of the genus with comments on P. californicum Nutt. Brittonia 49: 181-188.

Kuijt, J. 2003. Monograph of Phoradendron (Viscaceae). Syst. Bot. Monogr. 66: 1-643. 


\section{POST SCRIPTUM}

Cuando el artículo estaba en proceso editorial, gracias a las atenciones de los Bióls. Esteban Martínez y Rosalinda Medina, llegó al conocimiento de los autores la noticia de que en el estado de Morelos se había encontrado en 2010 una planta con características similares a las de P. perredactum. Poco tiempo antes de la entrega del número de la revista a la imprenta hemos recibido el material de herbario correspondiente y la comparación con el de Oaxaca indicó que se trata de la misma especie. La única diferencia observada es el color del fruto maduro, que en los ejemplares de Morelos es manifiestamente rojo-anaranjado, mientras que en los de Oaxaca invariablemente se vio café-morado. Es muy posible que esta aparente discrepancia obedece a las distintas épocas del año en que se obtuvieron las muestras.

Los datos de las plantas encontradas en el año 2010 son los siguientes:

México, Morelos, municipio de Cuernavaca, a $1.9 \mathrm{~km}$ al $\mathrm{N}$ de la entrada a Cuentepec, alt. $1508 \mathrm{~m}$, vegetación de selva baja caducifolia alterada. Hierba parásita de $1 \mathrm{~cm}$, flores naranja con verde y frutos naranja. 1.IV.2010. E. Martínez et al. 42151 (IEB, MEXU).

La fotografía tomada en el lugar de la colecta indica que el hospedero es una especie de Bursera de corteza exfoliante amarillenta, o sea del grupo de B. fagaroides (mismo que incluye también a B. discolor).

Este hallazgo sugiere que el taxon nuevo probablemente no es tan raro, pero que puede haber pasado inadvertido por su diminuto tamaño. Es de esperar su presencia también en los estados de Guerrero y de Puebla.

Los autores agradecen a Rosalinda Medina y a Esteban Martínez la gentileza recibida, así como el permiso de incluir en este lugar la información correspondiente, incluyendo la fotografía de la rama del árbol obtenida por los colectores en Morelos. 
Acta Botanica Mexicana 96: 3-10 (2011)
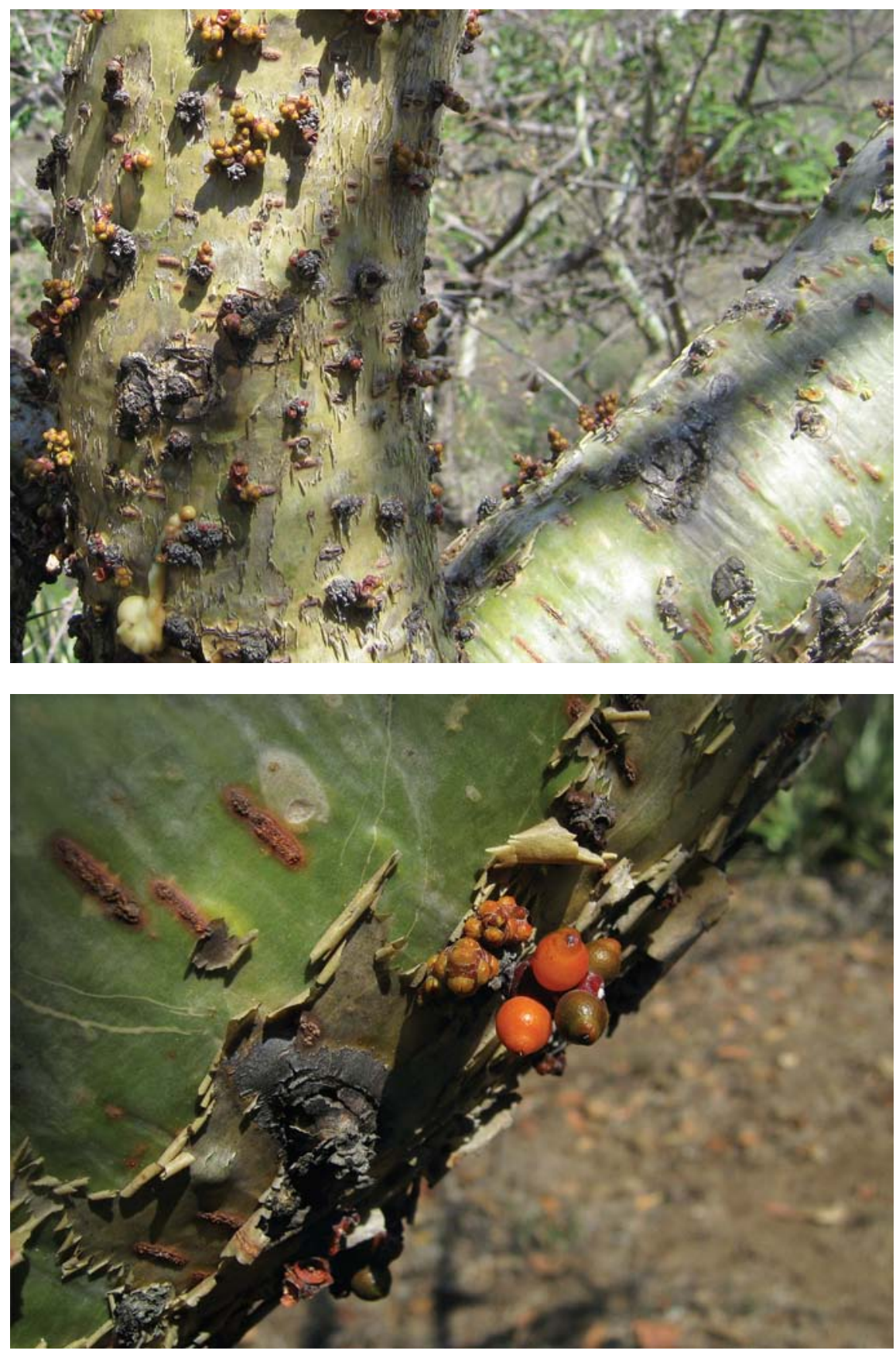

Phoradendron perredactum de Morelos. Fotografías de Esteban Martínez. 